Probability, Networks and Algorithms workload-dependent service rates

R. Bekker, S.C. Borst 
$\mathrm{CWI}$ is the National Research Institute for Mathematics and Computer Science. It is sponsored by the Netherlands Organization for Scientific Research (NWO).

$\mathrm{CWI}$ is a founding member of ERCIM, the European Research Consortium for Informatics and Mathematics.

CWI's research has a theme-oriented structure and is grouped into four clusters. Listed below are the names of the clusters and in parentheses their acronyms.

\section{Probability, Networks and Algorithms (PNA)}

Software Engineering (SEN)

Modelling, Analysis and Simulation (MAS)

Information Systems (INS)

Copyright (C) 2005, Stichting Centrum voor Wiskunde en Informatica

P.O. Box 94079, 1090 GB Amsterdam (NL)

Kruislaan 413, 1098 SJ Amsterdam (NL)

Telephone +31205929333

Telefax +31205924199

ISSN 1386-3711 


\title{
Optimal admission control in queues with workload- dependent service rates
}

\begin{abstract}
We consider a queueing system with a workload-dependent service rate. We specifically assume that the service rate is first increasing and then decreasing as a function of the amount of work. The latter qualitative behavior is quite common in practical situations, such as production systems. The admission of work into the system is controlled by a policy for accepting or rejecting jobs, depending on the state of the system. We seek an admission control policy that maximizes the long-run throughput. Under certain conditions, we show that a threshold policy is optimal, and derive a criterion for determining the optimal threshold value.
\end{abstract}

2000 Mathematics Subject Classification: 60K25; $90 \mathrm{~B} 22$

Keywords and Phrases: state-dependent rates; workload; throughput; arrival control; threshold policy; sample paths Note: The work of the first author was financially supported by a research grant from Philips Research. 



\title{
Optimal Admission Control in Queues with Workload-Dependent Service Rates*
}

\author{
René Bekker ${ }^{\dagger, \star}$ and Sem Borst ${ }^{\dagger, \star, \ddagger}$ \\ ${ }^{\dagger}$ Department of Mathematics \& Computer Science \\ Eindhoven University of Technology \\ P.O. Box 513, 5600 MB Eindhoven, The Netherlands \\ ${ }^{\star} \mathrm{CWI}$ \\ P.O. Box 94079, 1090 GB Amsterdam, The Netherlands \\ ${ }^{\ddagger}$ Bell Laboratories, Lucent Technologies \\ P.O. Box 636, Murray Hill, NJ 07974, USA
}

\begin{abstract}
We consider a queueing system with a workload-dependent service rate. We specifically assume that the service rate is first increasing and then decreasing as a function of the amount of work. The latter qualitative behavior is quite common in practical situations, such as production systems. The admission of work into the system is controlled by a policy for accepting or rejecting jobs, depending on the state of the system. We seek an admission control policy that maximizes the long-run throughput. Under certain conditions, we show that a threshold policy is optimal, and derive a criterion for determining the optimal threshold value.
\end{abstract}

\section{Introduction}

Queueing systems with state-dependent rates occur in many practical situations. A specific example concerns production systems where the productivity of the shop floor personnel depends on the level of work-in-process (workload). In particular, the productivity, i.e., the speed of the server, first increases when the workload is low until a certain optimum is attained and then decreases when the system reaches overload (caused by, e.g., stress factors), see for instance $[3,10]$. The latter qualitative behavior is quite characteristic of efficiency patterns observed in many practical scenarios.

In this paper, we consider an $\mathrm{M} / \mathrm{G} / 1$ queue with a service rate that is first increasing and then decreasing as a function of the workload. We assume that the speed of the server is intrinsically determined by the workload and the system characteristics and can thus not be directly controlled. However, there is an admission policy to control the amount of work present. Depending on the state of the system, arriving customers may be either accepted or rejected, or, equivalently, the facility may be either open or closed for potential

\footnotetext{
*The work of the first author was financially supported by a research grant from Philips Research.
} 
customers. The aim of this paper is to find the admission policy that maximizes the longrun throughput. In particular, under some assumption, we show that a threshold policy is optimal and give an intuitively appealing expression for the optimal threshold value.

The above-described M/G/1 queue with admission control may be modeled as a semiMarkov Decision Process (MDP). Most of the theory on MDP's concerns models with finite or countable state spaces. Because in the present queueing model both the admission policy and the service speed depend on the workload, we are dealing with an MDP with uncountable state space $[0, \infty)$. See for instance $[11,12,14]$ for some general MDP's with infinite state spaces. To derive structural properties of the optimal policy, a commonly used approach in MDP's is the construction of value functions that possess certain concavity properties. Because the value functions in our model typically do not exhibit such behavior, we apply sample-path techniques to compare different policies.

An interesting study showing several similarities with our model is [7]. In [7], the author considers an $\mathrm{M} / \mathrm{G} / 1$ queueing system with continuous-time arrival control and a fixed reward rate $R$ when the server is busy and holding cost rate $c x$ when the workload is $x$. Hence, such an M/G/1 queue can also be modeled as an MDP with the admission control depending on the system state, while the state space is infinite $[0, \infty)$. Using sample-path arguments and general theory on continuous-time MDP's developed in [6], the author proves the average-cost optimality of threshold policies.

Another branch of single-server queues with uncountable state spaces concerns $\mathrm{M} / \mathrm{G} / 1$ queues with service control. Specifically, the service speed may be continuously adapted based on the residual amount of work. In [5], the service speed equals $r_{1}$ when the workload is less than some fixed level $K$ and $r_{2}$ when the workload exceeds $K$. Under some fairly general cost functions, the author determines the optimal switching level $K$. In $[8,15]$, the server works at constant speed, but can be switched on and off. The cost function includes holding cost and switching cost for turning the server on. The average-cost optimality of $D$-policies is shown in $[8,15]$. In $D$-policies, the server is turned off only when the system becomes empty (and the server is on) and the server is turned on only when the workload exceeds level $D$ (and the server is off).

This paper is organized as follows: A detailed model description and several representations of the throughput are given in Section 2. In Section 3, the optimality of threshold policies under Assumption 2.1 (see Section 2) is shown. A criterion for the optimal threshold value is derived in Section 4. Several examples of (combinations of) service speed functions and service requirement distributions satisfying Assumption 2.1 are presented in Section 5. The optimal threshold value with corresponding throughput are explicitly determined in Section 6 for some special cases.

\section{Model description}

We consider an M/G/1 queue with a workload-dependent service rate. The customers (or jobs) arrive according to a Poisson process of rate $\lambda$. The service requirement of the $n$-th customer is $B_{n}, n=1,2, \ldots$, where the $B_{n}$ are assumed to be independent, identically distributed copies of a random variable $B$ with distribution $B(\cdot)$ and mean $\beta$. We also assume that the sequences of interarrival intervals and service requirements are independent.

The server works at a rate that depends on the amount of work in the system as described by some function $r(\cdot)$, i.e., the service rate is $r(x)$ when the amount of work is $x$. As in 
$[1,2,9]$, we assume that $r(0)=0$ and that $r(\cdot)$ is strictly positive, left-continuous, and has a right limit on $(0, \infty)$. In addition, we specifically focus on the case that $r(\cdot)$ is increasing on $\left(0, r_{\max }\right]$ and decreasing on $\left(r_{\max }, \infty\right)$ for some $r_{\max } \geq 0$.

The admission of work into the system is governed by a control policy which prescribes whether arriving customers are accepted or rejected, depending on the state of the system. We assume that the service requirement of a customer only becomes known after the acceptance decision, see Section 7 for a further discussion. Thus, the admission control policy may equivalently be interpreted as a rule for closing or opening access to the system. We seek an admission control policy that maximizes the long-run throughput. The longrun throughput under policy $\pi$ is defined as

$$
T H^{\pi}:=\lim _{t \rightarrow \infty} \frac{B^{\pi}(0, t)}{t},
$$

assuming the limit to exist. Here $B^{\pi}(0, t)$ denotes the amount of work completed during $[0, t]$ under policy $\pi$. A policy $\pi^{*}$ is said to be (strictly) optimal if $T H^{\pi^{*}} \geq T H^{\pi}\left(T H^{\pi^{*}}>\right.$ $T H^{\pi}$ ) for all policies $\pi \neq \pi^{*}$.

For now, we restrict the attention to the class of stationary and deterministic policies that base their actions on the current amount of work in the system only. For a given policy $\pi$, we use $\pi(x)=1$ to denote that it accepts a customer that arrives when the workload equals $x$ and write $\pi(x)=0$ otherwise. Later we will show that the found optimal policy is in fact optimal within a broader class that includes non-stationary and randomized policies as well.

Let $V_{t}^{\pi}$ be the workload at time $t$ and let $W_{n}^{\pi}$ be the workload just before the $n$-th arrival epoch. Denote by $V^{\pi}$ and $W^{\pi}$ the random variables with the corresponding steady-state distributions, if they exist, and let $v^{\pi}(\cdot)$ be the density of $V^{\pi}$.

We first consider the case $\lambda \beta<r_{\infty}$, with $r_{\infty}:=\lim _{x \rightarrow \infty} r(x)$. In that case, the system remains stable under the greedy policy that always accepts customers. Thus, the throughput achieved under the latter policy equals $\lambda \beta$, which is optimal, since the maximum achievable long-run throughput is bounded by the offered traffic load.

In the remainder of the paper we focus on the case $\lambda \beta>r_{\infty}$. (The boundary case $\lambda \beta=r_{\infty}$ is rather delicate, and a full analysis is beyond the scope of the present paper.) In that case, the system is unstable under the greedy policy that always accepts customers. Henceforth, we restrict the attention to policies $\pi$ such that $\pi(x)=0$ for all $x>M$ for some large $M$, which ensures the existence of the steady-state workload distribution. Even though the policy that always accepts customers may continue to be optimal, the maximum achievable throughput can be approached arbitrarily close for sufficiently large $M$.

Since the steady-state workload distribution exists, the throughput $T H^{\pi}$ under policy $\pi$ as defined above may in fact be expressed in several alternative ways. Observing that $B^{\pi}(0, t)=\int_{0}^{t} r\left(V_{u}^{\pi}\right) \mathrm{d} u$, the throughput may be equivalently written as

$$
T H^{\pi}=\lim _{t \rightarrow \infty} \frac{1}{t} \int_{0}^{t} r\left(V_{u}^{\pi}\right) \mathrm{d} u=\mathbb{E}\left[r\left(V^{\pi}\right)\right]=\int_{0}^{\infty} r(x) v^{\pi}(x) \mathrm{d} x .
$$

Invoking the further identity relation (with $A^{\pi}(0, t)$ denoting the amount of work accepted during $[0, t]$ under policy $\pi$ )

$$
B^{\pi}(0, t)=V_{0}^{\pi}+A^{\pi}(0, t)-V_{t}^{\pi}
$$


and noting that $V_{t}^{\pi} / t \rightarrow 0$ as $t \rightarrow \infty$, we observe that the throughput may also be expressed as

$$
T H^{\pi}=\beta \lim _{t \rightarrow \infty} \frac{N^{\pi}(0, t)}{t},
$$

where $N^{\pi}(0, t)$ denotes the number of accepted customers during $[0, t]$ under policy $\pi$. Using the PASTA property, the above expression may be further rewritten as

$$
T H^{\pi}=\lambda \beta \mathbb{P}\left(\pi\left(V^{\pi}\right)=1\right)=\lambda \beta\left(\pi(0) \mathbb{P}\left(V^{\pi}=0\right)+\int_{0}^{\infty} \pi(x) v^{\pi}(x) \mathrm{d} x\right) .
$$

Finally, we introduce some additional notation. Define

$$
R(x):=\int_{0}^{x} \frac{1}{r(y)} \mathrm{d} y, \quad 0<x<\infty,
$$

representing the time required for the system to empty in the absence of any arrivals, starting from workload $x$. In order to avoid technicalities, we assume that $R(x)<\infty$ for all $x>0$, as in [9]. Moreover, we assume that $\mathbb{E}[R(x+B+\delta)-R(x+B)] \rightarrow 0$, as $\delta \rightarrow 0$. The latter condition only rules out cases where the workload process is being absorbed in some positive workload level and is satisfied if, for instance, $r_{\infty}>0$ or if $B$ has finite support. Further define

$$
Z(x):=\int_{0}^{\infty} \int_{x}^{x+y} \frac{1}{r(z)} \mathrm{d} z \mathrm{~d} B(y)=\int_{x}^{\infty} \frac{1}{r(z)}(1-B(z)) \mathrm{d} z,
$$

representing the expected time required for the system to return to workload $x$ after a customer has been accepted, in the absence of any further arrivals. In the remainder of the paper, we make the following assumption with regard to $Z(x)$.

Assumption 2.1. There exists some $z_{\min } \geq 0$ such that $Z(x)$ is decreasing on $\left[0, z_{\min }\right]$ and increasing on $\left[z_{\min }, \infty\right)$.

The above assumption is satisfied for a wide class of M/G/1-type models with workloaddependent service rates. We give several illustrative examples in Section 5 .

To provide some intuition, suppose that the system operates according to the Last-Come First-Served Preemptive-Resume (LCFS-PR) discipline, which does not affect the workload process in any way. With that view in mind, $Z(x)$ may be thought of as the expected service time of a customer that arrives when the workload equals $x$, and $z_{\text {min }}$ represents the workload level at which arriving customers have the minimum expected service time. Thus, from the LCFS-PR perspective, the direct reward of accepting customers is first increasing (on $\left.\left(0, z_{\min }\right]\right)$ and then decreasing $\left(\right.$ on $\left.\left(z_{\min }, \infty\right)\right)$. However, the decision to either accept or reject also affects future rewards (service times). In Section 3, insights from the LCFS-PR discipline are applied to show that the optimal policy has a threshold stucture when Assumption 2.1 is satisfied.

\section{Optimality of threshold policies}

In the first part of this section, we only consider stationary deterministic policies. Since the actions of the admission control policy then only depend on the workload level $x$, we will also for brevity refer to the value of $x$ as the state of the system. An excursion from 
state $x$ is then a period that starts with the acceptance of a customer in state $x$ and ends with the first subsequent return to state $x$. For conciseness, we will frequently write that a policy accepts/rejects in an interval $[v, w]$ when it accepts/rejects customers that arrive when the workload is in the interval $[v, w]$. In the second part of this section, we show that the found optimal policy is in fact optimal within a broader class that also includes non-stationary and randomized policies.

Define $N^{\pi}(x)$ and $T^{\pi}(x)$ as follows:

$N^{\pi}(x) \equiv$ expected number of accepted customers during an excursion from state $x$ under policy $\pi$.

$T^{\pi}(x) \equiv$ expected duration of an excursion from state $x$ under policy $\pi$.

It may be verified that $N^{\pi}(x)$ and $T^{\pi}(x)$ are continuous, see also the proof of Lemma 3.2. Consider an arbitrary policy $\pi$ that rejects in $[x, x+\delta]$. Let $\pi^{\prime}$ be a modified policy, which does the same as $\pi$ except that it accepts in $[x, x+\delta]$. Let $G^{\pi}(y)$ be the expected number of excursions during a busy cycle that start from a workload level below $y$ under policy $\pi$, which are not part of an excursion starting from a level $z \in[x, y], y \geq x$.

Lemma 3.1. For some $\gamma \in(0,1)$, we have

$$
T H^{\pi^{\prime}}=(1-\gamma) T H^{\pi}+\gamma \frac{\int_{x}^{x+\delta} \beta N^{\pi^{\prime}}(y) \mathrm{d} G^{\pi^{\prime}}(y)}{\int_{x}^{x+\delta} T^{\pi^{\prime}}(y) \mathrm{d} G^{\pi^{\prime}}(y)} .
$$

Proof. By [13, Theorem 1], the throughput under policy $\pi$ may be equivalently expressed as $T H^{\pi}=\mathbb{E} R^{\pi} / \mathbb{E} T^{\pi}$, where $R^{\pi}$ is the reward (i.e., amount of work served) during a busy cycle and $T^{\pi}$ is the cycle length under policy $\pi$. Consider a busy cycle and take an arbitrary sample path of the workload process $\left\{V_{t}^{\pi^{\prime}}, t \geq 0\right\}$ under policy $\pi^{\prime}$. We construct a stochastic process $\hat{V}_{t}$ by deleting the excursions from level $y \in[x, x+\delta]$ and pasting together the remaining parts. First note that the residual interarrival time at a downcrossing of $y$ is still exponential (see, e.g., [2]). Now, it may be readily checked that $\hat{V}_{t}$ and $V_{t}^{\pi}$ have the same statistical properties. Thus, for the expected number of accepted customers during a busy cycle under policy $\pi^{\prime}, \mathbb{E} N^{\pi^{\prime}}$, we have

$$
\mathbb{E} N^{\pi^{\prime}}=\mathbb{E} N^{\pi}+\int_{x}^{x+\delta} N^{\pi^{\prime}}(y) \mathrm{d} G^{\pi^{\prime}}(y)
$$

and, equivalently, for the expected duration of a busy cycle

$$
\mathbb{E} T^{\pi^{\prime}}=\mathbb{E} T^{\pi}+\int_{x}^{x+\delta} T^{\pi^{\prime}}(y) \mathrm{d} G^{\pi^{\prime}}(y)
$$

Using Wald's theorem, we derive

$$
\begin{aligned}
\frac{\mathbb{E} R^{\pi^{\prime}}}{\mathbb{E} T^{\pi^{\prime}}} & =\frac{\beta \mathbb{E} N^{\pi^{\prime}}}{\mathbb{E} T^{\pi^{\prime}}}=\frac{\beta\left(\mathbb{E} N^{\pi}+\int_{x}^{x+\delta} N^{\pi^{\prime}}(y) \mathrm{d} G^{\pi^{\prime}}(y)\right)}{\mathbb{E} T^{\pi}+\int_{x}^{x+\delta} T^{\pi^{\prime}}(y) \mathrm{d} G^{\pi^{\prime}}(y)} \\
& =(1-\gamma) \frac{\mathbb{E} R^{\pi}}{\mathbb{E} T^{\pi}}+\gamma \frac{\int_{x}^{x+\delta} \beta N^{\pi^{\prime}}(y) \mathrm{d} G^{\pi^{\prime}}(y)}{\int_{x}^{x+\delta} T^{\pi^{\prime}}(y) \mathrm{d} G^{\pi^{\prime}}(y)}
\end{aligned}
$$

where $\gamma=\frac{\int_{x}^{x+\delta} T^{\pi^{\prime}}(y) \mathrm{d} G^{\pi^{\prime}}(y)}{\mathbb{E} T^{\pi}+\int_{x}^{x+\delta} T^{\pi^{\prime}}(y) \mathrm{d} G \pi^{\pi^{\prime}}(y)}$ represents the fraction of time spent on excursions starting between $x$ and $x+\delta$. This completes the proof.

Let $\pi^{*}$ denote an optimal policy, with corresponding throughput $T H^{*}=\mathbb{E}\left[r\left(V^{\pi^{*}}\right)\right]$. 


\section{Lemma 3.2. (Optimality properties)}

(i) it is strictly optimal to reject in $[v, w] \Longrightarrow \frac{\beta N^{\pi^{*}}(x)}{T^{\pi^{*}}(x)}<T H^{*}, \quad$ for almost every $x \in[v, w]$.

(ii) it is optimal to accept in $[v, w] \Longrightarrow \frac{\beta N^{\pi^{*}}(x)}{T^{\pi^{*}}(x)} \geq T H^{*}, \quad \forall x \in[v, w]$.

Note that the inequality in $(i)$ may hold with equality for some $x \in[v, w]$.

Proof. We first prove that

$$
\frac{\int_{x}^{x+\delta} N^{\pi^{\prime}}(y) \mathrm{d} G^{\pi^{\prime}}(y)}{\int_{x}^{x+\delta} T^{\pi^{\prime}}(y) \mathrm{d} G^{\pi^{\prime}}(y)} \rightarrow \frac{N^{\pi}(x)}{T^{\pi}(x)}, \quad \text { as } \delta \downarrow 0 .
$$

For some small $\delta>0$ and $y \in[x, x+\delta]$, we have

$$
T^{\pi^{\prime}}(y)-T^{\pi}(x+\delta) \leq(R(x+\delta)-R(y))\left(1+\lambda \max _{y \leq u \leq x+\delta} T^{\pi^{\prime}}(u)\right) \rightarrow 0, \quad \text { as } \delta \downarrow 0,
$$

where $R(x+\delta)-R(y)$ is the time required to go from $x+\delta$ to $y$ in the absence of any arrivals. Similarly, as $\delta \downarrow 0, T^{\pi^{\prime}}(y)-T^{\pi}(x+\delta)$ can be bounded from below by

$$
-\mathbb{E}[R(x+B+\delta)-R(y+B)]\left(1+\lambda \max _{y+B \leq u \leq x+B+\delta} \pi^{\prime}(u) T^{\pi^{\prime}}(u)\right) \rightarrow 0 .
$$

Applying similar arguments to $N^{\pi^{\prime}}(y)$ then yields (3). (Another way to see that (3) holds, is to observe that the density $\mathrm{d} G^{\pi}(\cdot)$ is well-defined.)

The remainder of the proof is by contradiction. For part (i), assume that the strictly optimal policy $\pi^{*}$ rejects in $[v, w]$, but there is some interval $(u, u+\delta) \subseteq[v, w]$, with $\delta>0$, such that $\beta N^{\pi^{*}}(x) / T^{\pi^{*}}(x) \geq T H^{*}$ for $x \in(u, u+\delta)$. Consider a modified policy $\pi$ which accepts in $[u, u+\delta]$ and follows $\pi^{*}$ otherwise. First using Lemma 3.1 and then letting $\delta \downarrow 0$ (and using (3)), it follows that $\mathbb{E}\left[r\left(V^{\pi}\right)\right] \geq T H^{*}$, contradicting the strict optimality of $\pi^{*}$. For part (ii), assume that the optimal policy $\pi^{*}$ accepts in $[v, w]$ but, for some $x \in[v, w]$, $\beta N^{\pi^{*}}(x) / T^{\pi^{*}}(x)<T H^{*}$. Using (3), it follows that there is some interval $U:=(u-\delta, u+$ $\delta) \subseteq[v, w]$ such that $\beta N^{\pi^{*}}(x) / T^{\pi^{*}}(x)<T H^{*}$ for every $x \in U$. Consider the modified policy $\pi$ that rejects in $U$ and follows $\pi^{*}$ otherwise. Using Lemma 3.1 (with $\pi^{\prime} \equiv \pi^{*}$ ), it is easily seen that $\mathbb{E}\left[r\left(V^{\pi}\right)\right]>T H^{*}$, contradicting the optimality of $\pi^{*}$.

Lemma 3.3. It is optimal to accept in $\left[0, z_{\min }\right]$.

Proof. It is obvious that it is optimal to accept in an empty system. Now, assume that it is not optimal to accept in $\left[0, z_{\min }\right]$. Then there is some policy $\pi$, such that $\pi(x)=1$ for $x \in\left[0, u^{*}\right]$, but $\pi(x)=0$ for $x \in\left(u^{*}, u^{*}+\delta\right]$, with $u^{*}+\delta \leq z_{\min }$ and $\delta>0$, that is strictly optimal.

Take some arbitrary $0<y<\delta$. In the proof, we compare $N^{\pi}\left(u^{*}\right)$ and $T^{\pi}\left(u^{*}\right)$ with $N^{\pi}\left(u^{*}+y\right)$ and $T^{\pi}\left(u^{*}+y\right)$. Using stochastic coupling, we show that $\beta N^{\pi}\left(u^{*}+y\right) / T^{\pi}\left(u^{*}+y\right)$ may be written as a combination of $\beta N^{\pi}\left(u^{*}\right) / T^{\pi}\left(u^{*}\right)$ and possibly contributions from some additional excursions. Since $\pi$ is assumed to be optimal, both terms provide an average 


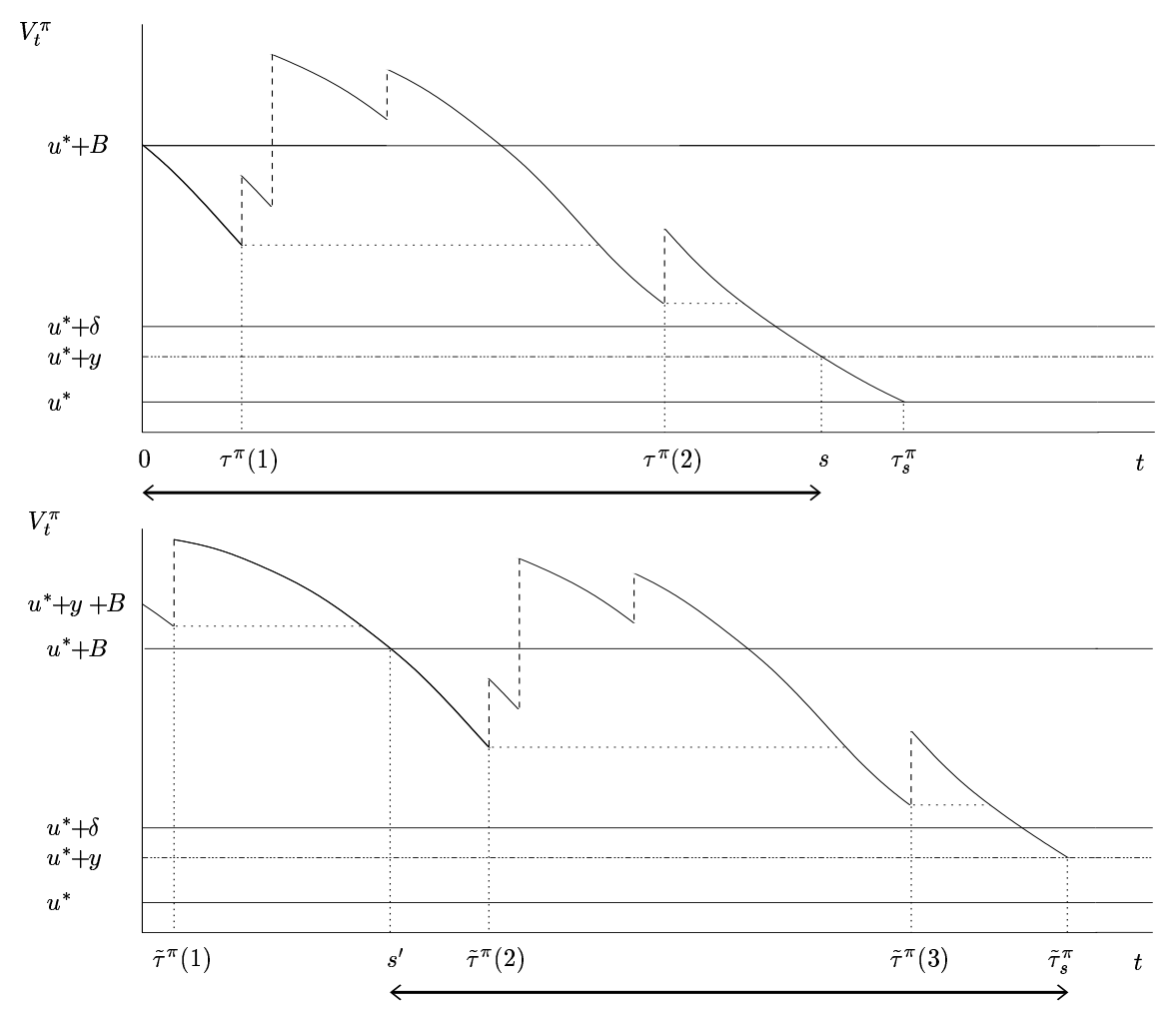

Figure 1: The sample paths of two excursions of $V_{t}^{\pi}$; one excursion from state $u^{*}$ and one excursion from state $u^{*}+y$. In this example $N=2$ and $M=3$.

reward of at least $T H^{*}$ by Lemma 3.2(ii). By Lemma 3.2(i), this contradicts the strict optimality of rejecting in $\left(u^{*}, u^{*}+\delta\right]$, because the coupling holds for any $y \in(0, \delta)$.

For the first part in the stochastic coupling, i.e., the part of the excursion from $u^{*}+y$ related to $\beta N^{\pi}\left(u^{*}\right) / T^{\pi}\left(u^{*}\right)$, observe that it follows from Assumption 2.1 that $Z\left(u^{*}\right) \geq Z\left(u^{*}+y\right)$, implying that the direct reward of accepting customers at level $u^{*}+y$ is at least as high as the direct reward of accepting at level $u^{*}$. For the second part, we use the fact that we only make additional excursions if they are advantageous.

First consider the expected duration of an excursion from level $u^{*}$ under policy $\pi$, and the expected number of accepted customers during such an excursion (i.e., $N^{\pi}\left(u^{*}\right)$ and $\left.T^{\pi}\left(u^{*}\right)\right)$. Let the first jump, initiating an excursion, occur at time 0 and observe that the workload level right after the first jump equals $u^{*}+B$, i.e., $V_{0^{+}}^{\pi}=u^{*}+B$. Note that the workload process attains local minima just before arrival instants at which customers are going to be accepted. Using terminology of random walks, define a stopping time $\tau_{s}^{\pi}:=\inf \left\{t \geq 0: V_{t}^{\pi} \leq u^{*}\right\}$, an equivalent notion measured in the number of arrivals $\tau^{\pi}:=$ $\inf \left\{k \geq 0: W_{k}^{\pi} \leq u^{*}\right\}$, and a sequence of descending ladder epochs $\tau^{\pi}(1)<\cdots<\tau^{\pi}(N)<$ $\tau^{\pi}$ with corresponding descending ladder heights $u^{*}+B>W_{\tau^{\pi}(1)}^{\pi}>\cdots>W_{\tau^{\pi}(N)}^{\pi}>u^{*}$, as follows: $\tau^{\pi}(1):=\inf \left\{0 \leq k \leq \tau^{\pi}: \pi\left(W_{k}^{\pi}\right)=1\right\}$, and for $n=2, \ldots, N\left(\right.$ if $\left.\tau^{\pi}(1)<\tau^{\pi}\right)$

$$
\tau^{\pi}(n+1):=\inf \left\{\tau^{\pi}(n)<k<\tau^{\pi}: W_{k}^{\pi}<W_{\tau^{\pi}(n)}^{\pi}, \pi\left(W_{k}^{\pi}\right)=1\right\} .
$$

Note that $W_{\tau^{\pi}(N)}^{\pi}>u^{*}+\delta$, since $\pi(x)=0$ for $x \in\left[u^{*}, u^{*}+\delta\right]$. A typical sample path in 
case $N=2$ is depicted in the first part of Figure 1. Using the above, we may write

$$
\begin{aligned}
& N^{\pi}\left(u^{*}\right)=1+\sum_{n=1}^{N} N^{\pi}\left(W_{\tau^{\pi}(n)}^{\pi}\right), \\
& T^{\pi}\left(u^{*}\right)=Z\left(u^{*}\right)+\sum_{n=1}^{N} T^{\pi}\left(W_{\tau^{\pi}(n)}^{\pi}\right) .
\end{aligned}
$$

Now consider $N^{\pi}\left(u^{*}+y\right)$ and $T^{\pi}\left(u^{*}+y\right)$. In this case, at time 0 the workload jumps to $u^{*}+y+B$, i.e., $V_{0^{+}}^{\pi}=u^{*}+y+B$. As defined above, we have a stopping time $\tilde{\tau}_{s}^{\pi}$, a discrete-time equivalent $\tilde{\tau}^{\pi}$, and a sequence of descending ladder epochs $0<\tilde{\tau}^{\pi}(1)<$ $\cdots<\tilde{\tau}^{\pi}(M)<\tilde{\tau}^{\pi}$ with corresponding descending ladder heights $u^{*}+y+B>W_{\tilde{\tau}^{\pi}(1)}^{\pi}>$ $\cdots>W_{\tilde{\tau}^{\pi}(M)}^{\pi}>u^{*}+y$ (see the second part of Figure 1 for a typical realization). Observe that the residual interarrival time at a downcrossing of $u^{*}+B$ is still exponential. Hence, using stochastic coupling and the fact that $W_{\tau^{\pi}(N)}^{\pi}>u^{*}+\delta$, the descending ladder epochs may be divided into two sets: (i) $\tilde{\tau}^{\pi}(1), \ldots, \tilde{\tau}^{\pi}(M-N)$ with $u^{*}+y+B>W_{\tilde{\tau}^{\pi}(1)}^{\pi}>\cdots>$ $W_{\tilde{\tau}^{\pi}(M-N)}^{\pi}>u^{*}+B$; and (ii) $\tilde{\tau}^{\pi}(M-N+1), \ldots, \tilde{\tau}^{\pi}(M)$ such that $W_{\tilde{\tau}^{\pi}(n+M-N)}^{\pi}={ }^{d} W_{\tau^{\pi}(n)}^{\pi}$ for $n=1, \ldots, N$. This coupling is illustrated in Figure 1 (with $N=2$ and $M=3$ ). In this figure, the sample paths in the range of the solid arrow (that is between $[0, s]$ and $\left[s^{\prime}, \tilde{\tau}_{s}^{\pi}\right]$ respectively) are identical. Using the arguments above, we have

$$
\begin{aligned}
& N^{\pi}\left(u^{*}+y\right)=1+\sum_{n=1}^{M-N} N^{\pi}\left(W_{\tilde{\tau}^{\pi}(n)}^{\pi}\right)+\sum_{n=1}^{N} N^{\pi}\left(W_{\tau^{\pi}(n)}^{\pi}\right), \\
& T^{\pi}\left(u^{*}+y\right)=Z\left(u^{*}+y\right)+\sum_{n=1}^{M-N} T^{\pi}\left(W_{\tilde{\tau}^{\pi}(n)}^{\pi}\right)+\sum_{n=1}^{N} T^{\pi}\left(W_{\tau^{\pi}(n)}^{\pi}\right) .
\end{aligned}
$$

Since $W_{\tilde{\tau}^{\pi}(n)}^{\pi}, n=1, \ldots, M-N$, are the workloads just before an arriving customer is accepted and $\pi$ is the supposed optimal policy, Lemma 3.2 yields

$$
\frac{\beta \sum_{n=1}^{M-N} N^{\pi}\left(W_{\tilde{\tau}^{\pi}(n)}^{\pi}\right)}{\sum_{n=1}^{M-N} T^{\pi}\left(W_{\tilde{\tau}^{\pi}(n)}^{\pi}\right)} \geq \beta \min _{n=1, \ldots, M-N} \frac{N^{\pi}\left(W_{\tilde{\tau}^{\pi}(n)}^{\pi}\right)}{T^{\pi}\left(W_{\tilde{\tau}^{\pi}(n)}^{\pi}\right)} \geq T H^{*} .
$$

Moreover, using (4) and (5) in addition to Assumption 2.1, we obtain

$$
\frac{\beta\left(1+\sum_{n=1}^{N} N^{\pi}\left(W_{\tau^{\pi}(n)}^{\pi}\right)\right)}{Z\left(u^{*}+y\right)+\sum_{n=1}^{N} T^{\pi}\left(W_{\tau^{\pi}(n)}^{\pi}\right)} \geq \frac{\beta N^{\pi}\left(u^{*}\right)}{T^{\pi}\left(u^{*}\right)} \geq T H^{*},
$$

where the second inequality relies on the fact that it is optimal to accept at level $u^{*}$. Combining (6)-(9) yields

$$
\begin{aligned}
& \frac{\beta N^{\pi^{\prime}}\left(u^{*}+y\right)}{T^{\pi^{\prime}}\left(u^{*}+y\right)} \\
& \quad \geq \min \left\{\frac{\beta \sum_{n=1}^{M-N} N^{\pi}\left(W_{\tilde{\tau}^{\pi}(n)}^{\pi}\right)}{\sum_{n=1}^{M-N} T^{\pi}\left(W_{\tilde{\tau}^{\pi}(n)}^{\pi}\right)}, \frac{\beta\left(1+\sum_{n=1}^{N} N^{\pi}\left(W_{\tau^{\pi}(n)}^{\pi}\right)\right)}{Z\left(u^{*}+y\right)+\sum_{n=1}^{N} T^{\pi}\left(W_{\tau^{\pi}(n)}^{\pi}\right)}\right\} \\
& \quad \geq T H^{*} .
\end{aligned}
$$

By Lemma 3.2 it can thus not be strictly optimal to reject at level $u^{*}+y, 0<y<\delta$. 
Theorem 3.1. There exists a threshold policy that is optimal among the class of stationary deterministic policies.

Proof. It follows from Lemma 3.3 that it is optimal to accept when the workload is in $\left[0, z_{\min }\right]$. Suppose that a threshold policy is not optimal, i.e., there exists some policy $\pi$ that is strictly better than any threshold policy. Let $n^{\pi}:=\int_{0}^{\infty} \max \left(\pi\left(x^{+}\right)-\pi(x), 0\right) \mathrm{d} x$ be the number of "gaps" of policy $\pi$, i.e., the number of times $\pi(\cdot)$ switches from 0 to 1 . Let $\pi$ be an optimal policy, which is strictly better than any threshold policy, with the least number of gaps, that is, $\pi=\arg \min _{\pi \in \Pi^{*}} n^{\pi}$, with $\Pi^{*}$ the class of optimal policies. This implies that there is some $u^{*}>z_{\min }$ and $\delta_{2}>\delta_{1}>0$ such that $\pi(x)=0$ on $\left(u^{*}, u^{*}+\delta_{1}\right)$ and $\pi(x)=1$ on $\left(u^{*}+\delta_{1}, u^{*}+\delta_{2}\right)$. We note that gaps consisting of singular points can be removed.

Take some arbitrary $0<y<\delta_{1}$. In the proof, we consider $N^{\pi}\left(u^{*}+y\right)$ and $T^{\pi}\left(u^{*}+y\right)$. Using the fact that it is optimal to accept in $\left(u^{*}+\delta_{1}, u^{*}+\delta_{2}\right)$, we show that $\beta N^{\pi}\left(u^{*}+\right.$ $y) / T^{\pi}\left(u^{*}+y\right) \geq T H^{*}$ (contradicting the fact that $\pi$ contains the least number of gaps among policies in $\left.\Pi^{*}\right)$. This follows from the fact that the direct reward of accepting at level $u^{*}+y$ exceeds the reward of accepting at any level $x>u^{*}+y$. Moreover, additional excursions are only made when they are advantageous.

Suppose that at time 0 an arriving customer with service requirement $B$ is accepted when the workload equals $u^{*}+y$, i.e., $V_{0^{+}}^{\pi}=u^{*}+y+B$. As in the proof of Lemma 3.3 (see also the first part of Figure 1, with $\delta_{1} \equiv \delta$ ), we may define "stopping times" $\tau_{s}^{\pi}$ and $\tau^{\pi}$ and a sequence of descending ladder epochs $\tau^{\pi}(1)<\cdots<\tau^{\pi}(N)<\tau^{\pi}$ with corresponding descending ladder heights $u^{*}+y+B>W_{\tau^{\pi}(1)}^{\pi}>\cdots>W_{\tau^{\pi}(N)}^{\pi}>u^{*}+y$. Note that $W_{\tau^{\pi}(N)}^{\pi}>u^{*}+\delta_{1}$ (if $N>0$ ), since $\pi(x)=0$ for $x \in\left[u^{*}, u^{*}+\delta_{1}\right]$. Applying this construction yields

$$
\begin{aligned}
& N^{\pi}\left(u^{*}+y\right)=1+\sum_{n=1}^{N} N^{\pi}\left(W_{\tau^{\pi}(n)}^{\pi}\right) \\
& T^{\pi}\left(u^{*}+y\right)=Z\left(u^{*}+y\right)+\sum_{n=1}^{N} T^{\pi}\left(W_{\tau^{\pi}(n)}^{\pi}\right) .
\end{aligned}
$$

By Lemma 3.2, $\beta \sum_{n=1}^{N} N^{\pi}\left(W_{\tau^{\pi}(n)}^{\pi}\right) / \sum_{n=1}^{N} T^{\pi}\left(W_{\tau^{\pi}(n)}^{\pi}\right) \geq T H^{*}$ since $\pi$ is assumed to be an optimal policy. Moreover, using a similar ladder height construction, it may be easily checked (in general) that

$$
\frac{N^{\pi}(x)}{T^{\pi}(x)} \leq \max _{v \geq x} \frac{1}{Z(v)}
$$

Hence, invoking Assumption 2.1 yields

$$
\frac{\beta}{Z\left(u^{*}+y\right)} \geq \frac{\beta}{Z\left(u^{*}+\delta_{1}\right)} \geq T H^{*} .
$$

Combining the above, we obtain $\beta N^{\pi}\left(u^{*}+y\right) / T^{\pi}\left(u^{*}+y\right) \geq T H^{*}$ for any $y \in\left(0, \delta_{1}\right)$. By Lemma 3.2, this contradicts the fact that policy $\pi$ has the minimum number of gaps among the class of optimal policies $\Pi^{*}$.

The ladder height construction in the proof of Theorem 3.1 allows us to generalize Relation (10): 
Proposition 3.1. For the throughput during an excursion from level $x$, we have the following bounds,

$$
\min _{v \geq x: \pi(v)=1} \frac{1}{Z(v)} \leq \frac{N^{\pi}(x)}{T^{\pi}(x)} \leq \max _{v \geq x: \pi(v)=1} \frac{1}{Z(v)} .
$$

These bounds are especially natural from the perspective of the LCFS-PR discipline. In that view, the proposition simply states that the throughput during an excursion from level $x$ is at least the minimum (and at most the maximum) of one over the mean service time of accepting at any level above $x$ if policy $\pi$ is applied.

Remark 3.1. The proof of Theorem 3.1 crucially depends on the fact that $Z(\cdot)$ has only one local minimum, i.e., Assumption 2.1. Suppose for the moment that $Z(\cdot)$ has L local minima. Thus, $Z(\cdot)$ is decreasing on $\left[z_{\max }^{k}, z_{\min }^{k}\right)$ and increasing on $\left[z_{\min }^{k}, z_{\max }^{k+1}\right), k=$ $1, \ldots, L$, where $z_{\max }^{1}=0$ and $z_{\max }^{L+1}=\infty$. Similar to the proof of Lemma 3.3, we deduce that if $\pi(x)=1$ for some $x \in\left[z_{\max }^{k}, z_{\min }^{k}\right)$, then $\pi(y)=1$ for all $y \in\left[x, z_{\min }^{k}\right)$ (note that $\pi(0)=1$ and accepting is thus optimal in $\left.\left[0, z_{\min }^{1}\right)\right)$. Also, it follows from the proof of Theorem 3.1 that if $\pi(x)=1$ for some $x \in\left[z_{\max }^{L}, \infty\right)$, then $\pi(y)=1$ for all $y \in\left[z_{\max }^{L}, x\right)$. However, the intervals $\left[z_{\min }^{k}, z_{\max }^{k+1}\right), k=1, \ldots, L-1$, are not covered by the proof. In particular, the trade-off between direct and future rewards remains undecided there.

Theorem 3.1 shows that the threshold policy is optimal among the class of stationary and deterministic policies. To prove that a (stationary and deterministic) threshold policy is also optimal within the broader class of policies considered in [13], we use insights from this section to construct an appropriate (value) function satisfying [13, Theorem 2]. The class of policies in [13] consists of all measurable decision rules, and includes non-stationary and non-deterministic policies.

Theorem 3.2. There exists a threshold policy that is optimal within the class of policies considered in [13].

Proof. Let $\pi$ be a threshold policy with threshold value $x^{*}$ that is optimal within the class of stationary and deterministic policies. Now, define $n^{\pi}(x)$ and $t^{\pi}(x)$ as follows:

$n^{\pi}(x) \equiv$ expected amount of work served in a period starting with workload level $x$ until the end of the busy cycle under policy $\pi$.

$t^{\pi}(x) \equiv$ expected length of a period starting with workload level $x$ until the end of the busy cycle under policy $\pi$.

Similar to [7], let

$$
\tilde{f}(x):=n^{\pi}(x)-T H^{\pi} t^{\pi}(x) .
$$

Consider $\mathbb{E}[\tilde{f}(x+B)]$ and divide the busy cycle in two parts; first we have an excursion from state $x$ followed by the remaining part of the cycle starting with a downcrossing of level $x$. Hence (see also [7, Lemma 6.3]),

$$
\mathbb{E}[\tilde{f}(x+B)]=\beta\left(N^{\pi}(x)-1\right)-T H^{\pi} T^{\pi}(x)+\tilde{f}(x),
$$

where the $N^{\pi}(x)-1$ stems from the fact that the arrival in state $x$ is not counted in $\mathbb{E}[\tilde{f}(x+B)]$.

Define, for $x \geq 0$,

$$
f(x):= \begin{cases}\beta+\mathbb{E}[\tilde{f}(x+B)], & \text { for } 0 \leq x \leq x^{*} \\ \tilde{f}(x), & \text { for } x>x^{*}\end{cases}
$$


where $x$ is the state of the system just before a decision epoch. By conditioning on the first arrival, we also obtain the following relationship between $\tilde{f}(\cdot)$ and $f(\cdot)$ :

$$
\tilde{f}(x)=\int_{0}^{\infty} f\left(R^{-1}\left((R(x)-y)^{+}\right)\right) \lambda e^{-\lambda y} \mathrm{~d} y-\frac{T H^{\pi}}{\lambda},
$$

with $R^{-1}(\cdot)$ the inverse function of $R(\cdot)$, see e.g. $[1,2,9]$ for details. Because $\pi$ is assumed to be an optimal stationary deterministic policy, Lemma 3.2 yields that $\beta N^{\pi}(x)-$ $T H^{\pi} T^{\pi}(x)$ is positive for $x \in\left[0, x^{*}\right)$, and non-positive for $x \in\left[x^{*}, \infty\right)$. Using the above in addition to (11) and (12), we obtain

$$
f(x)=\max \left\{\beta N^{\pi}(x)-T H^{\pi} T^{\pi}(x), 0\right\}+\tilde{f}(x) .
$$

Combining (11) with (13), we may rewrite (14) into

$$
\begin{gathered}
f(x)=\max \left\{\beta+\int_{0}^{\infty} \int_{0}^{\infty} f\left(R^{-1}\left((R(x+z)-y)^{+}\right)\right) \lambda e^{-\lambda y} \mathrm{~d} y \mathrm{~d} B(z),\right. \\
\left.\int_{0}^{\infty} f\left(R^{-1}\left((R(x)-y)^{+}\right)\right) \lambda e^{-\lambda y} \mathrm{~d} y\right\}-\frac{T H^{\pi}}{\lambda} .
\end{gathered}
$$

Thus the function $f(\cdot)$ satisfies the optimality equation for the average-cost criterion, i.e. Equation (3) in [13]. The theorem now follows directly from [13, Theorem 2].

\section{Criterion for the optimal threshold}

In Section 3 we showed that, if Assumption 2.1 is satisfied, a threshold policy is optimal. The derivation of that result also suggested the following criterion for the optimal threshold:

$$
T H^{\pi_{\bar{x}}}=\frac{\beta}{Z(\bar{x})},
$$

where $\pi_{\bar{x}}$ denotes a threshold policy with parameter $\bar{x}$. The above criterion is intuitively appealing when we consider marginal arguments. Informally speaking, the optimal threshold will be chosen such that the throughput just equals the expected reward of customers accepted in state $\bar{x}$ (which has reward $\beta / Z(\bar{x})$ ).

Moreover, the above criterion allows us to deduce some properties of the optimal threshold value. Using a similar construction as in (some of) the proofs of Section 3, it may be shown that $T H^{\pi_{\bar{x}}}$ is increasing as a function of $\lambda$. (To see this, we note that a higher $\lambda$ yields additional arrivals which are only accepted if the resulting excursions are advantageous.) Because $\beta / Z(\bar{x})$ is independent of $\lambda$ we can directly conclude from (15) that the optimal threshold value is decreasing in $\lambda$. It may also easily be checked that the optimal threshold approaches $z_{\min }$ as $\lambda \rightarrow \infty$. This behavior of the optimal threshold reveals the typical trade-off between direct and future rewards; the upper bound for the throughput is attained by accepting customers in state $z_{\min }$, but the optimal policy anticipates decreasing arrival rates by starting to accept customers at increasing workload levels to compensate for the increased probability of reaching an empty system (where the server is idle).

In the remainder of this section, we use another method to derive a criterion for the optimal threshold value and give some properties of $T H^{\pi_{\bar{x}}}$ as a function of $\bar{x}$. Moreover, 
when $Z(\cdot)$ does not satisfy Assumption 2.1, we show that a similar criterion as (15) holds for the optimal threshold value, which provides the optimal policy within the class of threshold policies. (Note that a threshold strategy may then not be optimal among the class of stationary and deterministic policies). However, we start with the general form of the throughput under a threshold strategy with some fixed threshold $\bar{x}$.

Observe that, for fixed $\bar{x}$, the workload under policy $\pi_{\bar{x}}$ has the same dynamics as an $\mathrm{M} / \mathrm{G} / 1$ queue with a general service rate and impatience of customers depending on the amount of work found upon arrival. Under policy $\pi_{\bar{x}}$ the model is in fact a special case of the finite-buffer queue in [2], with

$$
v^{\pi_{\bar{x}}}(x)= \begin{cases}\mathbb{P}\left(V^{\pi_{\bar{x}}}=0\right) K^{*}(x, 0), & 0<x \leq \bar{x}, \\ \mathbb{P}\left(V^{\pi_{\bar{x}}}=0\right)\left[K(x, 0)+\int_{0}^{\bar{x}} K(x, y) K^{*}(y, 0) \mathrm{d} y\right], & x>\bar{x},\end{cases}
$$

where $\mathbb{P}\left(V^{\pi_{\bar{x}}}=0\right)$ follows from normalization:

$$
\begin{aligned}
\mathbb{P}\left(V^{\pi_{\bar{x}}}=0\right)=[1+ & \int_{0}^{\bar{x}} K^{*}(x, 0) \mathrm{d} x+\int_{\bar{x}}^{\infty} K(x, 0) \mathrm{d} x \\
& \left.+\int_{\bar{x}}^{\infty} \int_{0}^{\bar{x}} K(x, y) K^{*}(y, 0) \mathrm{d} y \mathrm{~d} x\right]^{-1} .
\end{aligned}
$$

Here, the (iterated) kernels are defined as in [2,9]. That is, for $0 \leq y<x<\infty$, $K(x, y):=\lambda(1-B(x-y)) / r(x)$,

$$
K_{n+1}(x, y):=\int_{y}^{x} K(x, z) K_{n}(z, y) \mathrm{d} z
$$

and $K^{*}(x, 0):=\sum_{n=1}^{\infty} K_{n}(x, 0)$. Using the representation in (1) for the throughput, we obtain

$$
T H^{\pi_{\bar{x}}}=\lambda \beta \mathbb{P}\left(V^{\pi_{\bar{x}}}=0\right)\left(1+\int_{0}^{\bar{x}} K^{*}(x, 0) \mathrm{d} x\right) .
$$

Note that $Z(\bar{x})$ and $T H^{\pi_{\bar{x}}}$ are continuous and differentiable functions of $\bar{x}$. In order to determine the optimal threshold, it is useful to consider the derivative of $T H^{\pi_{\bar{x}}}$ with respect to $\bar{x}$.

Lemma 4.1. For the derivative of $T H^{\pi_{\bar{x}}}$, we have

$$
\frac{\mathrm{d}}{\mathrm{d} \bar{x}} T H^{\pi_{\bar{x}}}=\lambda \beta \mathbb{P}\left(V^{\pi_{\bar{x}}}=0\right) K^{*}(\bar{x}, 0)\left[1-T H^{\pi_{\bar{x}}} Z(\bar{x}) / \beta\right] .
$$

Proof. The proof is deferred to Appendix A.

Before we further discuss the optimal threshold criterion, we first derive some properties of $T H^{\pi_{\bar{x}}}$ as a function of $\bar{x}$. As in Lemma 3.1, consider a policy $\pi$ that does not accept in $[a, b]$ and a modified policy $\pi^{\prime}$, which does the same as $\pi$ except that $\pi^{\prime}(x)=1$ for $x \in[a, b]$. Then, the throughput under policy $\pi^{\prime}$ may be written as a convex combination of the throughput under policy $\pi$ and the throughput due to excursions starting from levels in $[a, b]$ (see Lemma 3.1). This relation is particularly useful in studying the relationship between $T H^{\pi_{\bar{x}}}$ and $Z(\cdot)$. 
Lemma 4.2. Suppose that (i) $\mathrm{d} Z(x) / \mathrm{d} x \leq 0$, for $x \in[a, b]$, and (ii) $T H^{\pi_{a}} \leq \beta / Z(a)$. Then,

$$
T H^{\pi_{x}} \leq \frac{\beta}{Z(x)}, \quad \text { for all } x \in[a, b] .
$$

If either (i) (for some $x \in[a, b]$ ) or (ii) holds with strict inequality, then (19) holds with strict inequality. Moreover, if the (strict) inequalities in (i) and (ii) are reversed, then the (strict) inequality in (19) is reversed.

Proof. Fix an arbitrary $x \in(a, b]$. Lemma 3.1 yields that, for $\gamma \in(0,1)$,

$$
T H^{\pi_{x}}=(1-\gamma) T H^{\pi_{a}}+\gamma \frac{\int_{a}^{x} \beta N^{\pi_{x}}(y) \mathrm{d} G^{\pi_{x}}(y)}{\int_{a}^{x} T^{\pi_{x}}(y) \mathrm{d} G^{\pi_{x}}(y)} .
$$

From (i) and Proposition 3.1, we obtain $\beta N^{\pi_{x}}(y) / T^{\pi_{x}}(y) \leq \beta / Z(x)$ for every $y \in[a, x]$. Invoking (ii), it trivially follows that

$$
T H^{\pi_{x}} \leq(1-\gamma) \frac{\beta}{Z(a)}+\gamma \frac{\beta}{Z(x)} \leq \frac{\beta}{Z(x)},
$$

where the last step is due to (i) again. Now, if (i) holds with strict inequality for some $x \in[a, b]$ then the second inequality of $(20)$ is strict, while the first one is strict if (ii) holds with strict inequality. The proof for the reversed signs is similar (use the lower bound in Proposition 3.1).

We now derive a criterion for the optimal threshold. Let $\pi_{t h}^{*}$ denote the optimal threshold strategy. Define the set $A:=\left\{x \geq 0: T H^{\pi_{x}}=\beta / Z(x)\right\}$. Note that, in general, $A$ is a collection of $N$ disjoint closed intervals $A_{i}, i=1, \ldots, N$, where each interval may be a singleton. However, if $A_{i}$ is not a singleton, then it follows directly from Lemma 4.2 that $Z(\cdot)$ is constant on $A_{i}$.

Proposition 4.1. If $A$ is the empty set, then the greedy policy is optimal and $T H^{\pi_{t h}^{*}}=r_{\infty}$. If $A$ is non-empty,

$$
T H^{\pi_{t h}^{*}}=\max \left\{r_{\infty}, \max _{x \in A} \beta / Z(x)\right\}
$$

where the greedy policy is optimal when $T H^{\pi_{t h}^{*}}=r_{\infty}$ and the optimal (finite) threshold is given by any $\bar{x} \in \arg \max _{x \in A} \beta / Z(x)$ otherwise.

Proof. For the threshold at 0 we have

$$
T H^{\pi_{0}}=\frac{\beta}{Z(0)+\lambda}<\frac{\beta}{Z(0)} .
$$

If $A$ is the empty set, then we have from the continuity of $Z(\cdot)$ and $T H^{\pi_{\bar{x}}}$ that $T H^{\pi_{x}}<$ $\beta / Z(x)$ for all $x$. Applying Lemma 4.1, we obtain that $\mathrm{d}\left(T H^{\pi_{x}}\right) / \mathrm{d} x>0$ for all $x$ and the greedy policy is thus optimal.

If $N>0$, then it follows from Lemma 4.1 that $A$ contains all points satisfying $\mathrm{d} T H^{\pi_{x}} / \mathrm{d} x=$ 0 . Hence, $A$ contains at least all extreme points. From (21) and Lemma 4.1 it follows that 0 is a local minimum. Moreover, $T H^{\pi_{x}} \rightarrow r_{\infty}$ as $x \rightarrow \infty$. Because $T H^{\pi_{\bar{x}}}$ is continuous, finding the global maximum of $T H^{\pi_{\bar{x}}}$ reduces to finding the maximum of $\beta / Z(x), x \in A$, and comparing it with $r_{\infty}$. 
Using Lemma 4.2 , some additional properties of $T H^{\pi_{\bar{x}}}$ as a function of $\bar{x}$ may be derived. For instance, it may be shown that if $Z(\cdot)$ has $m$ local maxima, then $N \leq 2 m-1$. In particular, if Assumption 2.1 is satisfied, then $N \leq 1$. This case is of special interest because a threshold policy is then optimal. Moreover, if in that case $N=1$, then $A \cap$ $\left[0, z_{\min }\right)$ is empty and each value in $A$ (possibly a singleton) is a global maximum of $T H^{\pi_{\bar{x}}}$. These arguments are summarized in the following corollary.

Corollary 4.1. Suppose that Assumption 2.1 is satisfied. If $A$ is the empty set, then the greedy policy is optimal. Otherwise, $N=1$ and $x \in A$ is an optimal threshold with corresponding throughput

$$
T H^{*}=\beta / Z(x) .
$$

Finally, if $r(x)$ is constant for $x \geq L$ (and Assumption 2.1 holds), there is an easy way to determine directly whether the greedy policy is optimal or not. From Lemma 4.2 we then deduce the following:

Corollary 4.2. Suppose Assumption 2.1 is satisfied and $r(x)=r_{\infty}$ for all $x \geq L$ for some $L>0$. Then, the greedy policy is optimal if and only if

$$
T H^{\pi_{L}} \leq \frac{\beta}{Z(L)}=r_{\infty}
$$

\section{Assumption on $Z(x)$}

Although Assumption 2.1 is quite natural, it involves the service-rate function as well as the distribution of the service requirement. Below, we give some examples satisfying this assumption, assuming that $r(\cdot)$ is increasing on $\left(0, r_{\max }\right]$ and decreasing on $\left(r_{\max }, \infty\right)$ for some $r_{\max } \geq 0$ (as described in Section 2). We consider both cases with general service requirement distributions and cases with a wide class of service-rate functions. In addition, we provide a natural example that does not have the desired properties. This case reveals the strong dependence on both the service-rate function and the service requirement distribution.

To show that Assumption 2.1 is satisfied, we frequently use the derivative of $Z(\cdot)$. Interchanging derivative and sum in addition to some rewriting, yields

$$
\begin{aligned}
\frac{\mathrm{d}}{\mathrm{d} x} Z(x) & =\int_{0}^{\infty} \frac{\mathrm{d}}{\mathrm{d} x}(R(x+b)-R(x)) \mathrm{d} B(b) \\
& =\int_{0}^{\infty} \frac{1}{r(x+b)} \mathrm{d} B(b)-\frac{1}{r(x)}=\int_{0}^{\infty} \frac{r(x)-r(x+b)}{r(x+b) r(x)} \mathrm{d} B(b) .
\end{aligned}
$$

For Assumption 2.1 to be satisfied, it remains to be shown that

$$
\begin{array}{ll}
r(x)-\mathbb{E}_{B}[r(x+B)] \leq 0, & x \in\left[0, z_{\min }\right] \\
r(x)-\mathbb{E}_{B}[r(x+B)] \geq 0, & x \in\left[z_{\min }, \infty\right) .
\end{array}
$$

Example 5.1. Suppose that $r_{\max }=0$, that is, $r(\cdot)$ is decreasing on the positive halfline. By definition, $r(y) \geq r(x)$, for $y>x$, and it is readily seen that Assumption 2.1 is satisfied. Also, $z_{\min }=0$ in this case. 
Example 5.2. Suppose that $B(x)=I(x \geq \beta)$, i.e., the service requirement is deterministic $\beta$. Observe that $\mathbb{E}_{B}[r(x+B)]$ is just the shifted $r(\cdot)$ function. Thus, $r(x) \leq \mathbb{E}_{B}[r(x+B)]$ if $x \in\left[0,\left(r_{\max }-\beta\right)^{+}\right]$and $r(x) \geq \mathbb{E}_{B}[r(x+B)]$ if $x \in\left[r_{\max }, \infty\right)$. Moreover, $r(x)$ is increasing on $\left[\left(r_{\max }-\beta\right)^{+}, r_{\max }\right]$, while $\mathbb{E}_{B}[r(x+B)]$ is decreasing on the same interval. This directly yields the required property.

Example 5.3. Suppose that $B(x)=1-e^{-\mu x}$, meaning that the service requirement is exponentially distributed. Observe that $r(x)-\mathbb{E}_{B}[r(x+B)] \geq 0$ for $x \geq r_{\max }$. Now take some arbitrary $x$ and $y$, with $0<x<y \leq r_{\max }$. Conditioning on the service requirement in case a customer arrives at level $x$ and using the memoryless property of the exponential distribution, we have

$$
\begin{aligned}
\mathbb{E}_{B}[r(x+B)] & =\int_{x}^{y} r(z) \mu e^{-\mu(z-x)} \mathrm{d} z+e^{-\mu(y-x)} \mathbb{E}_{B}[r(y+B)] \\
& \geq \int_{x}^{y} r(x) \mu e^{-\mu(z-x)} \mathrm{d} z+e^{-\mu(y-x)} \mathbb{E}_{B}[r(y+B)],
\end{aligned}
$$

and thus,

$$
r(x)-\mathbb{E}_{B}[r(x+B)] \leq e^{-\mu(y-x)} r(x)-e^{-\mu(y-x)} \mathbb{E}_{B}[r(y+B)] .
$$

Note that if $r(y)-\mathbb{E}_{B}[r(y+B)] \leq 0$, then $r(x)-\mathbb{E}_{B}[r(x+B)] \leq 0$ (since $r(y) \geq r(x)$ ). Similarly, if $r(x)-\mathbb{E}_{B}[r(x+B)] \geq 0$, then $r(y)-\mathbb{E}_{B}[r(y+B)] \geq 0$. This directly gives the desired property, where $z_{\min }=\arg \inf \left\{h: r(h) \geq \mathbb{E}_{B}[r(h+B)]\right\}$.

Example 5.4. Suppose that $r(\cdot)$ is defined as follows:

$$
r(x)= \begin{cases}r_{1}, & 0<x \leq a, \\ \text { increasing and concave, }, & a<x \leq r_{\max }, \\ \text { decreasing, } & x>r_{\max } .\end{cases}
$$

In addition, assume that $r_{\infty} \geq r_{1}$. From the properties of $r(\cdot)$, it is obvious that $r(x)-$ $\mathbb{E}_{B}[r(x+B)] \leq 0$ as $x \in(0, a]$ and $r(x)-\mathbb{E}_{B}[r(x+B)] \geq 0$ as $x \geq r_{\text {max }}$. Hence, $a \leq z_{\min } \leq r_{\max }$. Now take arbitrary $x, y$, with $a \leq x<y \leq r_{\max }$. First, consider the following:

$$
\begin{aligned}
\mathbb{E}_{B} & {[r(y+B)-r(x+B)] } \\
& =\int_{0}^{r_{\max }-x}(r(y+b)-r(x+b)) \mathrm{d} B(b)+\int_{r_{\max }-x}^{\infty}(r(y+b)-r(x+b)) \mathrm{d} B(b) \\
& \leq \int_{0}^{r_{\max }-x}(r(y)-r(x)) \mathrm{d} B(b)+0=(r(y)-r(x)) B\left(r_{\max }-x\right),
\end{aligned}
$$

where we used that $r(\cdot)$ is concave on $\left[a, r_{\max }\right]$ and decreasing on $\left[r_{\max }, \infty\right)$ in the second step. Using the above, we obtain

$$
\begin{aligned}
& r(x)-\mathbb{E}_{B}[r(x+B)] \\
& \quad=r(y)-\mathbb{E}_{B}[r(y+B)]+r(x)-r(y)+\mathbb{E}_{B}[r(y+B)]-\mathbb{E}_{B}[r(x+B)] \\
& \quad \leq r(y)-\mathbb{E}_{B}[r(y+B)]+(r(x)-r(y))\left(1-B\left(r_{\max }-x\right)\right) .
\end{aligned}
$$

As in Example 5.3, note that if $r(y)-\mathbb{E}_{B}[r(y+B)] \leq 0$, then $r(x)-\mathbb{E}_{B}[r(x+B)] \leq 0$ (since $r(y) \geq r(x))$. Similarly, if $r(x)-\mathbb{E}_{B}[r(x+B)] \geq 0$, then $r(y)-\mathbb{E}_{B}[r(y+B)] \geq 0$. Hence, Assumption 2.1 is satisfied, with $z_{\min }=\arg \inf \left\{h: r(h) \geq \mathbb{E}_{B}[r(h+B)]\right\}$. 
Finally, note that Example 5.1 is just a special case (take $a=r_{\max }=0$ ). However, we believe that Example 5.1 is a natural special case, which admits an easy verification of Assumption 2.1.

Example 5.5. Here we provide an example for which Assumption 2.1 is not satisfied. For simplicity, we choose specific values for some model parameters. A slightly more general model could be constructed by leaving some parameters unspecified, while leaving the structure unaltered.

Consider the following service rate function:

$$
r(x)= \begin{cases}r_{1}, & 0<x \leq a \\ (x-a) c+r_{1}, & a<x \leq r_{\max } \\ (\hat{h}-x) c+r_{1}, & r_{\max }<x \leq b, \\ r_{2}<r_{1}, & x>b,\end{cases}
$$

with $\hat{h}=2 r_{\max }-a$, implying that $r(\hat{h})=r_{1}$. Also, suppose that $B=a / 3$ with probability $1 / 2$ and $B=\hat{h}-a / 3$ with probability $1 / 2$, and take $c>3\left(r_{1}-r_{2}\right) / a$. After some calculations, we derive that $\mathrm{d} Z(x) / \mathrm{d} x$ is strictly positive on $\left(0, \frac{a}{3}\right)$ and $\left(\frac{2 a}{3}+\frac{r_{1}-r_{2}}{c}, \frac{4 a}{3}-\right.$ $\left.\frac{r_{1}-r_{2}}{c}\right)$ and strictly negative on $\left(\frac{a}{3}, \frac{2 a}{3}+\frac{r_{1}-r_{2}}{c}\right)$ and $\left(\frac{4 a}{3}-\frac{r_{1}-r_{2}}{c}, \infty\right)$. Clearly, $Z(\cdot)$ has two local minima and Assumption 2.1 is not satisfied in this case.

\section{Some examples}

In general, Expression (18) is suitable for a numerical calculation of the optimal threshold. Also, the characteristics of $T H^{\pi_{\bar{x}}}$ described in Section 4 suggest another numerical calculation of this optimal value, for instance, using a bisection method. In this section, we give some examples in which we obtain an analytically more tractable expression for the optimal throughput with corresponding optimal threshold value. In Subsection 6.1, we consider a two-level service rate: The service rate at time $t$ is $r_{1}$ when $V_{t}^{\pi} \leq a$ and $r_{2}<r_{1}$ when $V_{t}^{\pi} \geq a$ (see for instance [5]). In Subsection 6.2, we generalize the service rate to an arbitrary step function, but we restrict ourselves to exponential service requirements there.

In any case, if the greedy policy is not optimal, the optimal threshold value must satisfy Relation (15), see Proposition 4.1. Define

$$
z(\bar{x}):=\left[\mathbb{P}\left(V^{\pi_{\bar{x}}}=0\right)^{-1}-W(\bar{x}) \lambda Z(\bar{x})\right],
$$

where $W(x):=1+\int_{0}^{x} K^{*}(y, 0) \mathrm{d} y$ represents a non-normalized workload distribution. Using (18) and some straightforward manipulations, we may rewrite (15) into

$$
\frac{\mathbb{P}\left(V^{\pi_{\bar{x}}}=0\right)}{Z(\bar{x})} z(\bar{x})=0 .
$$

Note that both $\mathbb{P}\left(V^{\pi_{\bar{x}}}=0\right)>0$ and $Z(\bar{x})>0$ and finite. Finding the extremes of $T H^{\pi_{\bar{x}}}$ thus reduces to solving $z(\bar{x})=0$.

\subsection{Two-level service rate}

Suppose that the service rate is specified as

$$
r(x)= \begin{cases}r_{1}, & \text { for } 0<x \leq a \\ r_{2}, & \text { for } x>a\end{cases}
$$


where $0<r_{2}<r_{1}$. Define $\rho_{i}:=\lambda \beta / r_{i}, i=1,2$. Because the service-rate function is decreasing, we obtain from Example 5.1 that Assumption 2.1 is satisfied and a threshold policy is thus optimal. To determine the optimal threshold $\bar{x}$, we derive from Corollary 4.2 that we only need to consider $\bar{x} \leq a$.

Fix some $\bar{x} \in[0, a]$. Using results of $[2,9]$, the stationary workload distribution may be easily reduced to a more tractable expression. Let

$$
H(x):=\beta^{-1} \int_{0}^{x}(1-B(y)) \mathrm{d} y
$$

be the stationary residual service requirement distribution with density $h(\cdot)$. For $x \leq a$, $K(x, y)=\rho_{1} h(x-y)$ and it is well-known that $K^{*}(x, y)=\sum_{n=1}^{\infty} \rho_{1}^{n} h_{n}(x-y)$, where $h_{n}(\cdot)$ is the density of the $n$-fold convolution $H_{n}(\cdot)$ (see, e.g., $[2,9]$ ).

Now we determine the three elements on the right-hand side of (22) separately, after which we combine them to determine $z(\bar{x})$. First consider $\lambda Z(\bar{x})$. Using the definitions of $Z(\cdot)$ and $H(\cdot)$, respectively $(2)$ and $(23)$, yields

$$
\begin{aligned}
\lambda Z(\bar{x}) & =\lambda \int_{\bar{x}}^{a} \frac{1}{r_{1}}(1-B(x-\bar{x})) \mathrm{d} x+\lambda \int_{a}^{\infty} \frac{1}{r_{2}}(1-B(x-\bar{x})) \mathrm{d} x \\
& =\rho_{2}+\left(\rho_{1}-\rho_{2}\right) H(a-\bar{x}) .
\end{aligned}
$$

Second, consider the non-normalized workload distribution $W(\cdot)$. Interchanging integral and sum in addition to the results above, we immediately obtain for each $x \in[0, \bar{x}]$,

$$
W(x)=1+\int_{0^{+}}^{x} \sum_{n=1}^{\infty} \rho_{1}^{n} h_{n}(y) \mathrm{d} y=\sum_{n=0}^{\infty} \rho_{1}^{n} H_{n}(x) .
$$

Remark 6.1. Note that $W(\cdot) / W(a)$ is the steady-state workload distribution in a finite dam with speed $r_{1}$ and buffer size a. In case $\rho_{1}<1$, it is an easy exercise to see that the Laplace-Stieltjes transform of $W(\cdot)$ provides the well-known Pollaczek-Khinchine formula. If $\rho_{1} \geq 1, \int_{0}^{a} W(x) \mathrm{d} x$ is still finite and a steady-state workload distribution exists (see e.g. [9]). However, Cohen [4, 5] describes a more elegant way to determine $W(\cdot)$ in that case.

Finally, the first term of (22), that is the inverse of the normalizing constant $\mathbb{P}\left(V^{\pi_{\bar{x}}}=0\right)$, is the most complicated one. Using the expression for the steady-state workload density in addition to the results above, we derive for $\bar{x}<x \leq a$,

$$
\begin{aligned}
V^{\pi_{\bar{x}}}(x)= & V^{\pi_{\bar{x}}}(\bar{x})+\mathbb{P}\left(V^{\pi_{\bar{x}}}=0\right)\left[\int_{\bar{x}}^{x} \rho_{1} h(y) \mathrm{d} y\right. \\
& \left.\quad+\int_{\bar{x}}^{x} \int_{0^{+}}^{\bar{x}} \rho_{1} h(y-u) \sum_{n=1}^{\infty} \rho_{1}^{n} h_{n}(u) \mathrm{d} u \mathrm{~d} y\right] \\
= & \mathbb{P}\left(V^{\pi_{\bar{x}}}=0\right)\left[W(\bar{x})+\rho_{1}(H(x)-H(\bar{x}))\right. \\
& \left.\quad+\rho_{1} \int_{0^{+}}^{\bar{x}}(H(x-u)-H(\bar{x}-u)) \mathrm{d} W(u)\right] \\
= & \mathbb{P}\left(V^{\pi_{\bar{x}}}=0\right)\left[W(\bar{x})+\rho_{1} \int_{0^{-}}^{\bar{x}}(H(x-u)-H(\bar{x}-u)) \mathrm{d} W(u)\right],
\end{aligned}
$$


where we used $W(0)=1$ in the final step. Note that, for $x>a, K(x, y)=\rho_{2} h(x-y)$. Using similar arguments, we obtain for $x>a$,

$$
\begin{aligned}
V^{\pi_{\bar{x}}}(x)=V^{\pi_{\bar{x}}}(a)+\mathbb{P}\left(V^{\pi_{\bar{x}}}=0\right)\left[\int_{a}^{x} \rho_{2} h(y) \mathrm{d} y\right. & \\
& \left.\quad+\int_{a}^{x} \int_{0^{+}}^{\bar{x}} \rho_{2} h(y-u) \sum_{n=1}^{\infty} \rho_{1}^{n} h_{n}(u) \mathrm{d} u \mathrm{~d} y\right] \\
= & \mathbb{P}\left(V^{\pi_{\bar{x}}}=0\right)\left[W(\bar{x})+\rho_{1} \int_{0^{-}}^{\bar{x}}(H(a-u)-H(\bar{x}-u)) \mathrm{d} W(u)\right. \\
& \left.\quad+\rho_{2} \int_{0^{-}}^{\bar{x}}(H(x-u)-H(a-u)) \mathrm{d} W(u)\right] .
\end{aligned}
$$

By (25), we have $\rho_{1} \int_{0}^{\bar{x}} H(\bar{x}-u) \mathrm{d} W(u)=W(\bar{x})-1$. Letting $x \rightarrow \infty$ and some rewriting then yields

$$
\mathbb{P}\left(V^{\pi_{\bar{x}}}=0\right)^{-1}=\rho_{2} W(\bar{x})+\left(\rho_{1}-\rho_{2}\right) \int_{0^{-}}^{\bar{x}} H(a-u) \mathrm{d} W(u)+1 .
$$

It is now easy to get $z(\bar{x})$. Substituting (24)-(26) into (22) gives

$$
z(\bar{x})=1+\left(\rho_{2}-\rho_{1}\right)\left[W(\bar{x}) H(a-\bar{x})-\int_{0^{-}}^{\bar{x}} H(a-u) \mathrm{d} W(u)\right] .
$$

Summarizing, Corollary 4.2 implies that the greedy policy is optimal if and only if

$$
\rho_{2}-\left(\rho_{2}-\rho_{1}\right) W(a)>0 .
$$

Otherwise, $T H^{*}=\rho /\left(\rho_{2}+\left(\rho_{1}-\rho_{2}\right) H\left(a-x^{*}\right)\right)$, with $\rho:=\lambda \beta$ and $x^{*}$ is a solution to $z\left(x^{*}\right)=0$.

In general, the convolution in (27) can only be determined numerically. However, if the service requirement follows a phase-type distribution, explicit expressions can be obtained. For instance, if $B(x)=1-e^{-\mu x}$ (see also Subsection 6.2), then after quite lengthy but standard calculations, it follows that, for $\rho_{1} \neq 1$,

$$
z(\bar{x})=1+\frac{\rho_{2}-\rho_{1}}{\rho_{1}-1} e^{-\mu a}\left(e^{\mu \bar{x}}-e^{\mu \rho_{1} \bar{x}}\right) .
$$

In case $\rho_{1}=1$, we obtain

$$
z(\bar{x})=1-\left(\rho_{2}-1\right) \mu \bar{x} e^{-\mu(a-\bar{x})} .
$$

\subsection{Exponential service requirements}

Suppose that the service requirements are exponentially distributed with mean $1 / \mu$, i.e., $1-B(x)=e^{-\mu x}$. Then, for fixed $\bar{x}$, the steady-state workload density is given in $[2$, Corollary 7.1]:

$$
v^{\pi_{\bar{x}}}(x)=\frac{\lambda \mathbb{P}\left(V^{\pi_{\bar{x}}}=0\right)}{r(x)} \exp \{-\mu x+\lambda R(x \wedge \bar{x})\},
$$

where $\mathbb{P}\left(V^{\pi_{\bar{x}}}=0\right)$ follows from normalization. In this subsection, we also assume that the service rate is a step function. More specifically, let $r(x)=r_{i}$ for $x \in\left[a_{i-1}, a_{i}\right)$, 
$i=1, \ldots, N$ (where $a_{0}=0$ ), and let $r(x)=r_{N+1}<r_{N}$ for $x \geq a_{N}$. Denote $\rho_{i}=\lambda /\left(\mu r_{i}\right)$, $i=1, \ldots, N+1$ and assume for simplicity that $\rho_{i} \neq 1$.

Example 5.3 shows that Assumption 2.1 is satisfied and a threshold policy is thus optimal. By Corollary 4.2, either the greedy policy is optimal, or the optimal threshold $x^{*}$ is less than $a_{N}$. Let $\bar{x} \in\left[a_{n}, a_{n+1}\right)$ for some $n \leq N-1$. Next, we consider each of the three elements of $z(\bar{x})$ separately, after which we combine them into an expression for $x^{*}$ satisfying (15). However, for later use, we first define the following three constants. (In the sequel we follow the convention that empty sums are equal to 0 .)

$$
\begin{aligned}
\gamma_{n} & =\exp \left\{\sum_{k=1}^{n}\left(\frac{\lambda}{r_{k}}-\frac{\lambda}{r_{k+1}}\right) a_{k}\right\} \\
C_{n} & =\frac{1}{1-\rho_{1}}+\sum_{k=1}^{n}\left(\frac{\rho_{k}}{\rho_{k}-1}-\frac{\rho_{k+1}}{\rho_{k+1}-1}\right) \gamma_{k-1} e^{-\mu\left(1-\rho_{k}\right) a_{k}}, \\
D_{n} & =\sum_{k=n+1}^{N}\left(\rho_{k+1}-\rho_{k}\right) e^{-\mu a_{k}} .
\end{aligned}
$$

First, consider $\lambda Z(\bar{x})$. Using (2) and rewriting the integral, we obtain

$$
\begin{aligned}
\lambda Z(\bar{x})= & \int_{\bar{x}}^{a_{n+1}} \frac{\lambda}{r_{n+1}} e^{-\mu(x-\bar{x})} \mathrm{d} x+\sum_{k=n+1}^{N-1} \int_{a_{k}}^{a_{k+1}} \frac{\lambda}{r_{k+1}} e^{-\mu(x-\bar{x})} \mathrm{d} x \\
& \quad+\int_{a_{N}}^{\infty} \frac{\lambda}{r_{N+1}} e^{-\mu(x-\bar{x})} \mathrm{d} x \\
= & \rho_{n+1}\left(1-e^{-\mu\left(a_{n+1}-\bar{x}\right)}\right)+\sum_{k=n+1}^{N-1} \rho_{k+1}\left(e^{-\mu\left(a_{k}-\bar{x}\right)}-e^{-\mu\left(a_{k+1}-\bar{x}\right)}\right) \\
& +\rho_{N+1} e^{-\mu\left(a_{N}-\bar{x}\right)} \\
= & \rho_{n+1}+D_{n} e^{\mu \bar{x}} .
\end{aligned}
$$

Second, consider $W(\cdot)$, i.e., the workload distribution 'without normalization'. It is easily checked that the time to empty the system starting from $a_{i}, i=1, \ldots, n$, in the absence of any arrivals (i.e., $\left.R\left(a_{i}\right)\right)$ equals $a_{i} / r_{i}+\sum_{k=1}^{i-1}\left(1 / r_{k}-1 / r_{k+1}\right) a_{k}$. Hence, for $x \in\left[a_{i}, a_{i+1}\right)$, we may deduce that

$$
\exp \{\lambda R(x)\}=\exp \left\{\frac{\lambda\left(x-a_{i}\right)}{r_{i+1}}+\lambda R\left(a_{i}\right)\right\}=\gamma_{i} \exp \left\{\frac{\lambda x}{r_{i+1}}\right\} .
$$


Now, for $i=1, \ldots, n$, using (29) and (31), we obtain after some standard algebra, that

$$
\begin{aligned}
V^{\pi_{\bar{x}}} & \left(a_{i}\right) / \mathbb{P}\left(V^{\pi_{\bar{x}}}=0\right) \\
& =1+\sum_{k=0}^{i-1} \int_{a_{k}}^{a_{k+1}} \frac{\lambda}{r_{k+1}} e^{-\mu x+\lambda R(x)} \mathrm{d} x \\
& =1+\sum_{k=0}^{i-1} \frac{\rho_{k+1}}{\rho_{k+1}-1} \gamma_{k}\left(e^{-\mu\left(1-\rho_{k+1}\right) a_{k+1}}-e^{-\mu\left(1-\rho_{k+1}\right) a_{k}}\right) \\
& =\frac{1}{1-\rho_{1}}+\sum_{k=0}^{i-1} \frac{\rho_{k+1}}{\rho_{k+1}-1} \gamma_{k} e^{-\mu\left(1-\rho_{k+1}\right) a_{k+1}}-\sum_{k=1}^{i-1} \frac{\rho_{k+1}}{\rho_{k+1}-1} \gamma_{k} e^{-\mu\left(1-\rho_{k+1}\right) a_{k}} \\
& =\frac{1}{1-\rho_{1}}+\sum_{k=1}^{i} \frac{\rho_{k}}{\rho_{k}-1} \gamma_{k-1} e^{-\mu\left(1-\rho_{k}\right) a_{k}}-\sum_{k=1}^{i-1} \frac{\rho_{k+1}}{\rho_{k+1}-1} \gamma_{k-1} e^{-\mu\left(1-\rho_{k}\right) a_{k}} \\
& =C_{i-1}+\frac{\rho_{i}}{\rho_{i}-1} \gamma_{i-1} e^{-\mu\left(1-\rho_{i}\right) a_{i}},
\end{aligned}
$$

where we used $\gamma_{k} e^{\mu \rho_{k+1} a_{k}}=\gamma_{k-1} e^{\mu \rho_{k} a_{k}}$ in the fourth equality. Thus, combining (29) and (31) with the above, we obtain, after similar manipulations,

$$
\begin{aligned}
V^{\pi_{\bar{x}}}(\bar{x}) & =V^{\pi_{\bar{x}}}\left(a_{n}\right)+\mathbb{P}\left(V^{\pi_{\bar{x}}}=0\right) \int_{a_{n}}^{\bar{x}} \frac{\lambda}{r_{n+1}} e^{-\mu x+\lambda R(x)} \mathrm{d} x \\
& =V^{\pi_{\bar{x}}}\left(a_{n}\right)+\mathbb{P}\left(V^{\pi_{\bar{x}}}=0\right) \frac{\rho_{n+1}}{\rho_{n+1}-1} \gamma_{n}\left(e^{-\mu\left(1-\rho_{n+1}\right) \bar{x}}-e^{-\mu\left(1-\rho_{n+1}\right) a_{n}}\right) \\
& =\mathbb{P}\left(V^{\pi_{\bar{x}}}=0\right)\left[C_{n}+\frac{\rho_{n+1}}{\rho_{n+1}-1} \gamma_{n} e^{-\mu\left(1-\rho_{n+1}\right) \bar{x}}\right],
\end{aligned}
$$

which completes the calculation of $W(\cdot)$ (since $\left.W(\bar{x}) \mathbb{P}\left(V^{\pi_{\bar{x}}}=0\right)=V^{\pi_{\bar{x}}}(\bar{x})\right)$.

For the first term on the right-hand side of $(22)$, i.e., $\mathbb{P}\left(V^{\pi_{\bar{x}}}=0\right)^{-1}$, we use similar arguments as for the previous one. We first consider $V^{\pi_{\bar{x}}}(x)$ with $x>a_{n+1}$ and let $i=\arg \max \left\{a_{i}: a_{i} \leq x\right\}$ be the largest $a_{i}$ smaller than $x$. Using (29) and applying (31) to determine $\lambda R(\bar{x})$, we obtain after similar algebra as above, that

$$
\begin{aligned}
V^{\pi_{\bar{x}}}(x)=V^{\pi_{\bar{x}}}(\bar{x})+ & \gamma_{n} e^{\mu \rho_{n+1} \bar{x}} \mathbb{P}\left(V^{\pi_{\bar{x}}}=0\right)\left[\int_{\bar{x}}^{a_{n+1}} \frac{\lambda}{r_{n+1}} e^{-\mu y} \mathrm{~d} y\right. \\
& \left.+\sum_{k=n+1}^{i-1} \int_{a_{k}}^{a_{k+1}} \frac{\lambda}{r_{k+1}} e^{-\mu y} \mathrm{~d} y+\int_{a_{i}}^{x} \frac{\lambda}{r_{i+1}} e^{-\mu y} \mathrm{~d} y\right] \\
=\mathbb{P}\left(V^{\pi_{\bar{x}}}=\right. & 0)\left[C_{n}+\frac{\rho_{n+1}^{2}}{\rho_{n+1}-1} \gamma_{n} e^{-\mu\left(1-\rho_{n+1}\right) \bar{x}}\right. \\
& \left.+\gamma_{n} e^{\mu \rho_{n+1} \bar{x}} \sum_{k=n+1}^{i}\left(\rho_{k+1}-\rho_{k}\right) e^{-\mu a_{k}}-\gamma_{n} \rho_{i+1} e^{-\mu x+\mu \rho_{n+1} \bar{x}}\right] .
\end{aligned}
$$

Thus, letting $x \rightarrow \infty$, we obtain the normalizing constant:

$$
\mathbb{P}\left(V^{\pi_{\bar{x}}}=0\right)^{-1}=C_{n}+\frac{\rho_{n+1}^{2}}{\rho_{n+1}-1} \gamma_{n} e^{-\mu\left(1-\rho_{n+1}\right) \bar{x}}+\gamma_{n} D_{n} e^{\mu \rho_{n+1} \bar{x}} .
$$


The function $z(\bar{x})$ can now easily be rewritten into a more appealing expression. In particular, substituting (30), (34), and $W(\bar{x})$ resulting from (33) into (22) and some reordering of terms, yields

$$
\begin{aligned}
z(\bar{x})= & \left(1-\rho_{n+1}\right) C_{n}+D_{n} \gamma_{n} e^{\mu \rho_{n+1} \bar{x}} \\
& -D_{n} e^{\mu \bar{x}}\left(C_{n}+\frac{\rho_{n+1}}{\rho_{n+1}-1} \gamma_{n} e^{-\mu\left(1-\rho_{n+1}\right) \bar{x}}\right) \\
= & \left(1-\rho_{n+1}\right) C_{n}-C_{n} D_{n} e^{\mu \bar{x}}-\frac{D_{n} \gamma_{n}}{\rho_{n+1}-1} e^{\mu \rho_{n+1} \bar{x}}
\end{aligned}
$$

Solving $z(\bar{x})=0$ is thus remarkably simple in this case, since the variable $\bar{x}$ only appears in two of the exponents. Summarizing, we conclude that the optimal policy is of the threshold type where the optimal threshold value is given by the solution of $z(\bar{x})=0$. Moreover $T H^{*}=\beta / Z(\bar{x})$, where $\lambda Z(\bar{x})$ is given in $(30)$.

Remark 6.2. It is easily checked that, in case $N=1$, the formula for $z(\bar{x})$ indeed reduces to (28).

\section{Concluding remarks and further research}

In the present paper, we considered the problem of optimal admission control in a system with a workload-dependent service rate. We assumed that the service requirement only becomes known right after the decision of accepting or rejecting jobs. Our objective was to find a policy that maximizes the long-run throughput. Under some assumptions (in particular Assumption 2.1), we showed that a threshold policy for accepting jobs is optimal and derived a criterion for the optimal threshold value.

We note that our main assumption, i.e. Assumption 2.1, involves sufficient conditions for optimality of threshold policies. An interesting subject for further research is to examine the structure of the optimal policy when Assumption 2.1 is not satisfied.

Moreover, there are various interesting model variations. For instance, the analysis is significantly changed if information about the service requirement is available. In that case, the decision will not only depend on the workload level, but also on the size of the job, yielding a two-dimensional state space. A characterization of the optimal policy in that model might be a subject of further study. We note that a threshold policy will not be optimal in general. However, in some special cases, as for, e.g., deterministic service requirements or decreasing service rate functions, the optimal policy continues to be of the threshold type.

Other model variations are systems where jobs can be partly accepted (or rejected). The simplest version concerns a model where an infinite amount of work becomes available at Poisson instants and the policy prescribes the amount of work to accept. In some sense, this model is related to the case $\lambda \rightarrow \infty$ in the model of the present paper, which may be interpreted as an infinite supply of jobs and the policy prescribes the time to accept a new job. More interesting are systems where the supply of work is bounded by the service requirements of arriving jobs and the decision is the amount of work to accept. In that case, the state space is two-dimensional and the action space is continuous. The structure of the optimal policy in the latter model is also left for future investigation. 


\section{A Proof of Lemma 4.1}

Lemma 4.1 For the derivative of $T H^{\pi_{\bar{x}}}$, we have

$$
\frac{\mathrm{d}}{\mathrm{d} \bar{x}} T H^{\pi_{\bar{x}}}=\lambda \beta \mathbb{P}\left(V^{\pi_{\bar{x}}}=0\right) K^{*}(\bar{x}, 0)\left[1-T H^{\pi_{\bar{x}}} Z(\bar{x}) / \beta\right] .
$$

Proof. We first consider $\mathbb{P}\left(V^{\pi_{\bar{x}}}=0\right)$. Observe that the double integration in (16) may be equivalently expressed as follows: $\int_{x=\bar{x}}^{\infty} \int_{y=0}^{\bar{x}}=\int_{y=0}^{\bar{x}} \int_{x=y}^{\infty}-\int_{x=0}^{\bar{x}} \int_{y=0}^{x}$. Using the definition of $K^{*}$, interchanging integral and sum and finally applying (17), we may write

$$
\begin{aligned}
\int_{0}^{\bar{x}} K(\bar{x}, y) K^{*}(y, 0) \mathrm{d} y & =\sum_{n=1}^{\infty} \int_{0}^{\bar{x}} K(\bar{x}, y) K_{n}(y, 0) \mathrm{d} y \\
& =\sum_{n=0}^{\infty} K_{n+1}(\bar{x}, 0)-K_{1}(\bar{x}, 0) \\
& =K^{*}(\bar{x}, 0)-K(\bar{x}, 0) .
\end{aligned}
$$

Taking the derivative of $\mathbb{P}\left(V^{\pi_{\bar{x}}}=0\right)$ with respect to $\bar{x}$, we obtain from (16) and the reordering of integration that

$$
\begin{aligned}
& \frac{\mathrm{d}}{\mathrm{d} \bar{x}} \mathbb{P}\left(V^{\pi_{\bar{x}}}=0\right) \\
& =-\mathbb{P}\left(V^{\pi_{\bar{x}}}=0\right)^{2}\left(K^{*}(\bar{x}, 0)-K(\bar{x}, 0)\right. \\
& \left.+\int_{\bar{x}}^{\infty} K(x, \bar{x}) K^{*}(\bar{x}, 0) \mathrm{d} x-\int_{0}^{\bar{x}} K(\bar{x}, y) K^{*}(y, 0) \mathrm{d} y\right) \\
& =-\mathbb{P}\left(V^{\pi_{\bar{x}}}=0\right)^{2} K^{*}(\bar{x}, 0) \int_{\bar{x}}^{\infty} K(x, \bar{x}) \mathrm{d} x,
\end{aligned}
$$

where we used (35) in the second step. Now, invoking (18) and taking the derivative of $T H^{\pi_{\bar{x}}}$ with respect to $\bar{x}$ yields

$$
\begin{aligned}
\frac{\mathrm{d}}{\mathrm{d} \bar{x}} T H^{\pi_{\bar{x}}}= & \lambda \beta \mathbb{P}\left(V^{\pi_{\bar{x}}}=0\right) K^{*}(\bar{x}, 0)+\lambda \beta \frac{\mathrm{d}}{\mathrm{d} \bar{x}} \mathbb{P}\left(V^{\pi_{\bar{x}}}=0\right)\left(1+\int_{0}^{\bar{x}} K^{*}(x, 0) \mathrm{d} x\right) \\
= & \lambda \beta \mathbb{P}\left(V^{\pi_{\bar{x}}}=0\right) K^{*}(\bar{x}, 0) \\
& \times\left[1-\mathbb{P}\left(V^{\pi_{\bar{x}}}=0\right) \int_{\bar{x}}^{\infty} K(x, \bar{x}) \mathrm{d} x\left(1+\int_{0}^{\bar{x}} K^{*}(x, 0) \mathrm{d} x\right)\right] \\
= & \lambda \beta \mathbb{P}\left(V^{\pi_{\bar{x}}}=0\right) K^{*}(\bar{x}, 0)\left[1-T H^{\pi_{\bar{x}}} Z(\bar{x}) / \beta\right],
\end{aligned}
$$

where the final step follows from (18) and the fact that $\lambda Z(\bar{x})=\int_{\bar{x}}^{\infty} K(x, \bar{x}) \mathrm{d} x$. This completes the proof.

\section{Acknowledgment}

The authors are grateful to Prof. J.W.M. Bertrand for posing questions that motivated the present work and to Dr. Phil Whiting for stimulating discussions. 


\section{References}

[1] Bekker, R., S.C. Borst, O.J. Boxma, O. Kella (2004). Queues with workload-dependent arrival and service rates. Queueing Systems 46, 537-556.

[2] Bekker, R. (2005). Finite-buffer queues with workload-dependent service and arrival rates. Queueing Systems 50, 231-253.

[3] Bertrand, J.W.M., H.P.G. van Ooijen (2002). Workload based order release and productivity: a missing link. Production Planning \& Control 13, 665-678.

[4] Cohen, J.W. (1976). On Regenerative Processes in Queueing Theory. Lecture Notes in Economics and Mathematical Systems 121. Springer-Verlag, Berlin.

[5] Cohen, J.W. (1976). On the optimal switching level for an M/G/1 queueing system. Stochastic Processes and Their Applications 4, 297-316.

[6] Doshi, B.T. (1974). Continuous time control of Markov processes on an arbitrary state space. Ph.D. thesis, Cornell University.

[7] Doshi, B.T. (1977). Continuous time control of the arrival process in an M/G/1 queue. Stochastic Processes and Their Applications 5, 265-284.

[8] Feinberg, E.A., O. Kella (2002). Optimality of $D$-policies for an M/G/1 queue with a removable server. Queueing Systems 42, 355-376.

[9] Harrison, J.M., S.I. Resnick (1976). The stationary distribution and first exit probabilities of a storage process with general release rule. Mathematics of Operations Research 1, 347-358.

[10] Van Ooijen, H.P.G., J.W.M. Bertrand (2003). The effects of a simple arrival rate control policy on throughput and work-in-process in production systems with workload dependent processing rates. International Journal of Production Economics 85, 61-68.

[11] Ritt, R.K., L.I. Sennott (1992). Optimal stationary policies in general state space Markov decision chains with finite action sets. Mathematics of Operations Research 17, 901-909.

[12] Ross, S.M. (1968). Arbitrary state Markov decision processes. The Annals of Mathematical Statistics 39, 2118-2122.

[13] Ross, S.M. (1970). Average cost semi-Markov decision processes. Journal of Applied Probability 7, 649-656.

[14] Schäl, M. (1993). Average optimality in dynamic programming with general state space. Mathematics of Operations Research 18, 163-172.

[15] Tijms, H.C. (1976). Optimal control of the workload in an M/G/1 queueing system with removable server. Math. Operationsforsch. Statist. 7, 933-944. 